\title{
MEWS++: Enhancing the Prediction of Clinical Deterioration in Admitted Patients through a Machine Learning Model
}

\author{
Arash Kia ${ }^{1}$, Prem Timsina ${ }^{1}$, Himanshu N. Joshi ${ }^{1}{ }^{\mathbb{D}}$, Eyal Klang ${ }^{2}$, Rohit R. Gupta ${ }^{3}$, \\ Robert M. Freeman ${ }^{1}\left(\mathbb{D}\right.$, David L Reich ${ }^{4}$, Max S Tomlinson ${ }^{5,6}$, Joel T Dudley ${ }^{5,6}$, \\ Roopa Kohli-Seth ${ }^{3}$, Madhu Mazumdar ${ }^{1}$ (D) and Matthew A Levin ${ }^{1,4,5,6, *(D)}$ \\ 1 Institute for Healthcare Delivery Science, Department of Population Health Science and Policy, Icahn School \\ of Medicine at Mount Sinai, New York, NY 10029, USA; arash.kia@mssm.edu (A.K.); \\ prem.timsina@mssm.edu (P.T.); himanshu.joshi@mssm.edu (H.N.J.); \\ Robert.Freeman@mountsinai.org (R.M.F.); madhu.mazumdar@mountsinai.org (M.M.) \\ 2 Department of Diagnostic Imaging, The Chaim Sheba Medical Center at Tel HaShomer, Sackler Faculty of \\ Medicine, Tel Aviv University, Ramat Gan 52662, Israel; Eyal.Klang@mountsinai.org \\ 3 Institute for Critical Care Medicine, Icahn School of Medicine at Mount Sinai, New York, NY 10029, USA; \\ rohit.gupta@mountsinai.org (R.R.G.); roopa.kohli-seth@mountsinai.org (R.K.-S.) \\ 4 Department of Anesthesiology, Perioperative and Pain Medicine, Icahn School of Medicine at Mount Sinai, \\ New York, NY 10029, USA; david.reich@mountsinai.org \\ 5 Department of Genetics and Genomics Sciences, Icahn School of Medicine at Mount Sinai, New York, \\ NY 10029, USA; max.tomlinson@mssm.edu (M.S.T.); joel.dudley@mssm.edu (J.T.D.) \\ 6 Icahn Institute for Data Science and Genomic Technology, Icahn School of Medicine at Mount Sinai, \\ New York, NY 10029, USA \\ * Correspondence: matthew.levin@mssm.edu; Tel.: +212-241-8382
}

Received: 25 December 2019; Accepted: 17 January 2020; Published: 27 January 2020

\begin{abstract}
Early detection of patients at risk for clinical deterioration is crucial for timely intervention. Traditional detection systems rely on a limited set of variables and are unable to predict the time of decline. We describe a machine learning model called MEWS++ that enables the identification of patients at risk of escalation of care or death six hours prior to the event. A retrospective single-center cohort study was conducted from July 2011 to July 2017 of adult (age > 18) inpatients excluding psychiatric, parturient, and hospice patients. Three machine learning models were trained and tested: random forest (RF), linear support vector machine, and logistic regression. We compared the models' performance to the traditional Modified Early Warning Score (MEWS) using sensitivity, specificity, and Area Under the Curve for Receiver Operating Characteristic (AUC-ROC) and Precision-Recall curves (AUC-PR). The primary outcome was escalation of care from a floor bed to an intensive care or step-down unit, or death, within $6 \mathrm{~h}$. A total of 96,645 patients with 157,984 hospital encounters and 244,343 bed movements were included. Overall rate of escalation or death was $3.4 \%$. The RF model had the best performance with sensitivity $81.6 \%$, specificity $75.5 \%$, AUC-ROC of 0.85 , and AUC-PR of 0.37 . Compared to traditional MEWS, sensitivity increased $37 \%$, specificity increased $11 \%$, and AUC-ROC increased $14 \%$. This study found that using machine learning and readily available clinical data, clinical deterioration or death can be predicted $6 \mathrm{~h}$ prior to the event. The model we developed can warn of patient deterioration hours before the event, thus helping make timely clinical decisions.
\end{abstract}

Keywords: Failure to Rescue; Modified Early Warning Score; clinical deterioration; Machine Learning Classifiers; Unexpected Escalation 


\section{Introduction}

Timely detection of patient deterioration and prompt clinical intervention is key to lowering the potentially preventable morbidity and mortality among hospital inpatients [1,2]. All too often however, the early abnormalities and clinical signs that precede serious clinical deterioration may remain unidentified [3,4]. Studies on the identification of hospital patients at risk for clinical deterioration over last two decades have resulted in the development of prediction tools that use rule-based scoring [5-8]. The Modified Early Warning Score (MEWS), for example, incorporates physiological parameters including systolic blood pressure, pulse rate, respiratory rate, temperature, and level of consciousness. There are some limitations to this approach: (1) the schemas of these scores are usually defined manually; (2) alarm triggers rely on empirically chosen values; (3) the thresholds are usually set to capture the greatest percentage of clinically significant events, resulting in non-specific alerts that include a large number of false alarms. This creates an excess of warning notifications that can generate alarm fatigue [9-11]. Indeed, it has been shown that as the number of non-actionable alarms increases, the response time of providers increases as well [12]. Additionally, the usefulness of these systems is limited by inability to quantify the risk for decompensation and the lack of a defined time window for intervention.

Recent work by Bedoya et al. has confirmed the poor performance and minimal impact of implementing a traditional Early Warning Score [13]. However, machine learning approaches that use large Electronic Health Record (EHR) data can be trained to have good performance in predicting deterioration, exceeding that of traditional models $[14,15]$. We hypothesized that a machine learning model trained on a large dataset could have better performance than MEWS. We aimed for our model to predict escalation of care or death within the next $6 \mathrm{~h}$. A six-hour prediction window was chosen based on clinical considerations such as the typical duration of nursing and physician shift length $(8-12 \mathrm{~h})$ and the desire to alert within a time frame that was both believable and actionable by the same care team that received the alert.

\section{Patients and Methods}

Institutional Research Board approval was obtained for this retrospective cohort study. Inclusion criteria were all adult inpatient admissions (age > 18) between July 2011 and July 2017. We excluded patients admitted to psychiatry, labor and delivery, and hospice units. This is due to low frequency of escalation or lack of adequate monitoring in these units. Patients were categorized into Major Diagnostic Categories (MDCs) derived from ICD-9 diagnostic codes [16].

We retrieved data via our institutional data warehouse from the following sources: admission-discharge-transfer (ADT) events; structured clinical assessments (e.g., nursing notes); physiologic data (e.g., vital signs); laboratory results; and automated electrocardiogram (ECG) results.

\subsection{Phenotyping of Patient Deterioration}

Most existing MEWS algorithms have been developed on cohorts of modest size (i.e., dozens to hundreds of patients). Adverse events were identified via either retrospective manual chart review or prospective data collection $[6,17,18]$. Given our intention to use a much larger cohort, we developed an automated phenotyping algorithm to identify escalation of care [19]. We decided a priori to base our algorithm on bed movement (ADT) data. Our base assumption was that bed movements were independent events. Using administrative data, each bed in the hospital was assigned to a generalized level of care such as floor, ICU, step-down, and so-on. We then applied a set of rules developed by authors MAL and RF to classify each bed transition as expected or unexpected (see Table S1 in the Supplement for the full list of phenotyping rules). Expected transitions were those between beds at the same level of care. Unexpected transitions were those to a higher level of care such as the ICU or step-down unit, or death. 
A retrospective chart review was performed to validate the performance of the phenotyping algorithm. A random sample of 286 hospital encounters (1193 bed movements) was drawn from 157,984 hospital encounters. Authors MAL and RF reviewed the charts in consensus and classified each as a true positive, false positive, true negative or false negative. The review was done in rounds of $20-40$ charts to tune the phenotyping algorithm. The final result showed sensitivity of $90.5 \%$, specificity of $81.9 \%$, and positive predictive value (PPV) of $82.1 \%$.

\subsection{Algorithms Evaluated}

We compared a Random Forest (RF) algorithm to two additional machine learning algorithms: a linear Support Vector Machine (SVM) and Logistic Regression (LR). RF is a classifier that fits a number of decision trees on sub-samples of the dataset and uses averaging to improve the predictive accuracy and control over-fitting [20]. SVM is a classifier that attempts to maximize the linear distance between $p$-dimensional vectors representing instances of each class, where $p$ is the number of features $[21,22]$.

\subsection{Defining Optimal Prediction Time}

The time of escalation (unexpected transition) or death was defined as $t_{0}$. For patients who never had an escalation of care (negative cases), the time of discharge was used as $t_{0}$. Times of predictions, $t_{p}$, were defined as the time prior to $t_{0}$ at which predictions were generated (Figure 1). Predictions were generated every two hours prior to the escalation event, with data sampled from the 24-h period prior to prediction (Figure 1). A time series was created by defining a sampling window as the 24-h period before the prediction time $t_{p}$. Then, data were sampled every $4 \mathrm{~h}$ within the window, i.e., six times in $24 \mathrm{~h}$ (Figure 1). This frequency reflects the typical interval between vital sign measurements on a medical-surgical hospital unit. The result was a time series $V=\left\{V_{1}, V_{2}, \ldots V_{6}\right\}$ with 6 measurements per 24-h sampling window for each feature. Missing values were imputed by using the median value of the variable over the entire cohort at the sampling time point [23].

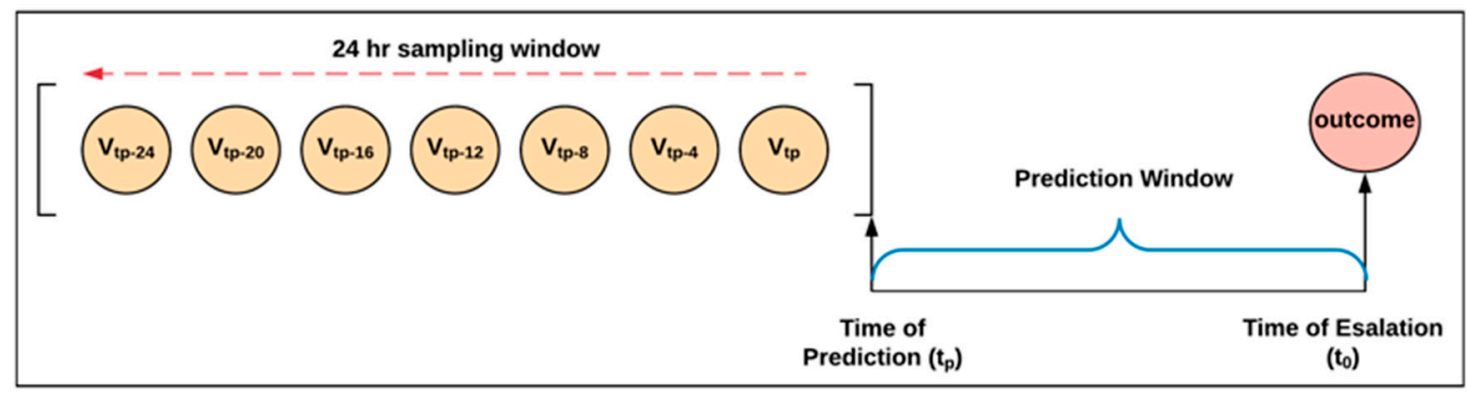

Figure 1. Prediction time and sampling window. $t_{0}$ is the time of escalation, death, or discharge for patients with no event. Prediction time $t_{p}$ is the time prior to $t_{0}$ at which a prediction was generated. The sampling window is the 24 -h period preceding the prediction time $t_{p}$.

Performance of the models were compared, and an optimal prediction time of $6 \mathrm{~h}$ was chosen based on clinical and operational considerations such as anticipated impact on nursing and clinician workload.

\subsection{Data Encoding and Scaling}

Categorical variables were one-hot-encoded. Continuous variables were scaled using the MLLib min-max scaler to be within the range $(0,1)[24]$.

\subsection{Resampling}

The overall rate of unexpected events (escalation of care) was under $5 \%$, versus $95 \%$ expected events. This large variance between the prevalence of the two classes can lead to over-fitting and under-performance [25]. We applied random under-sampling to remove instances of the majority class (expected events) until both classes were equally balanced. 


\subsection{Calculation of MEWS Score}

MEWS score was calculated as described by Subbe et al. [6]. Briefly, four physiologic variables (systolic blood pressure, pulse rate, temperature, and respiratory rate) and one level of consciousness assessment were given a numeric weighting of $0-3$ with the MEWS score calculated as the additive sum of all 5 sub-scores. A MEWS score of 2 was used as the cut-off based on the literature and by visual inspection of the sensitivity and specificity curves [6] (Supplementary Figure S1).

\section{Model Development}

All models were built using Python v.2.7.13, MLlib, and Spark 2.3 [26]. Plotly 2.0.6 was used for visualizations. The primary model developed was the RF model. We called this model MEWS++. We also evaluated the linear SVM and LR models. The default probability threshold of 0.5 was used for all models. Parameters for the models are listed in Supplementary Table S2. Briefly, the RF model used 500 trees, max depth of 10 and max bin of 32. Both SVM and LR models used 10 folds and 500 iterations. The regularization parameter for the SVM model was 0.1 and for the LR model it was 0.3. The Spark implementation of linear SVM has no slack parameter.

Feature selection and model tuning were performed on a training set consisting of $70 \%$ of the bed movement data. Ten-fold cross-validation was used for both feature selection and model development. The $F_{1}$ score was used for best model selection. $F_{1}$ is the harmonic average of the precision (PPV) and recall (sensitivity), calculated as:

$$
F_{1}=2 * \frac{\text { precision } * \text { recall }}{\text { precision }+ \text { recall }}
$$

\subsection{Feature Selection}

Recursive feature elimination (RFE) was used as the feature selection approach. Feature selection was only performed only on the training set. A list of 89 variables was created based on review of relevant literature and clinician feedback, and were used to build an initial RF model (Supplementary Table S3). Then, using the Gini coefficient, the least important features were pruned [27]. All features were recursively tested in this fashion. The final list of variables chosen by RFE is listed in Supplementary Table S4.

\subsection{Model Training}

The final reduced feature set selected by RFE was then used to build a model, and this model was trained and cross-validated only on the training set. An average $F_{1}$ score was computed for each internal validation model. The final model chosen for testing was the one with the largest average $\mathrm{F}_{1}$ score.

Under-sampling may result in loss of potentially useful information for defining the majority class, and thereby can compromise the model accuracy. In order to rule out such influence on RF/MEWS++ model performance, we performed 10 iterations of under-sampling on the majority class in the training data and compared the performance of resultant models with the main RF-based model. The results are shown in Supplementary Table S5. The standard deviations of all performance metrics ranged from 0.003 to 0.67 , indicating that the final chosen RF model was not affected by under-sampling bias.

\subsection{External Model Testing}

The remaining thirty percent $(30 \%)$ of the bed movement data were used exclusively as an independent test set for the final chosen RF model. PPV (precision), sensitivity (recall), specificity, $\mathrm{F}_{1}$ score and the Area Under the Receiver-Operator Curve (AUC) and Area Under the Precision-Recall Curve (AUPRC) were used as metrics. 


\section{Results}

The raw cohort consisted of 96,645 patients with 157,984 hospital encounters and 244,343 bed movements. Under-sampling resulted in a training cohort of 15,818 bed movements. The test cohort consisted of 102,066 bed movements. Basic demographics of the training and test cohorts are shown in Table 1 . The mean age was 63.4 years. Approximately half of the population was female $(50.1 \%)$. The rate of unexpected escalation of care or death in the validation set was $3.4 \% .56 .8 \%$ escalated to an ICU, $41.9 \%$ to telemetry, $1.3 \%$ escalated to an intermediate care unit, and $0.7 \%$ died. There was a significant variation of unexpected escalation rate seen between MDC categories (Supplementary Figure S2). The respiratory, infectious, and circulatory groups showed the highest rates of escalation $(9.2 \%, 5.9 \%$, and $4.5 \%$, respectively).

Table 1. Cohort demographics.

\begin{tabular}{|c|c|c|c|c|c|}
\hline & & Total N (\%) & Training (\%) & Test (\%) & $p$-Value \\
\hline $\begin{array}{c}\text { Bed } \\
\text { movements }\end{array}$ & & 117,884 & 15,818 & 102,066 & \\
\hline $\begin{array}{c}\text { Bed } \\
\text { movements per } \\
\text { encounter }\end{array}$ & & $1.67 \pm 1.15$ & $1.33 \pm 0.76$ & $1.59 \pm 0.99$ & \\
\hline $\begin{array}{l}\text { Unique } \\
\text { Patients * }\end{array}$ & & 63,100 & 13,168 & 58,742 & \\
\hline Age & $\begin{array}{c}18-45 \\
45-65 \\
65-80 \\
>80\end{array}$ & $\begin{array}{l}19,422(16.5) \\
40,942(34.7) \\
37,596(31.9) \\
19,924(16.9)\end{array}$ & $\begin{array}{l}2107(13.3) \\
5060(32.0) \\
5266(33.3) \\
3385(21.4)\end{array}$ & $\begin{array}{l}17,315(17.0) \\
35,882(35.2) \\
32,330(31.7) \\
16,539(16.2)\end{array}$ & $<0.001$ \\
\hline Gender & $\begin{array}{l}\text { Female } \\
\text { Male } \\
\text { Other }\end{array}$ & $\begin{array}{c}58,345(49.5) \\
59,532(50.5) \\
7(0.0)\end{array}$ & $\begin{array}{c}7760(49.1) \\
8057(50.9) \\
1(0.0)\end{array}$ & $\begin{array}{c}50,585(49.6) \\
51,475(50.4) \\
6(0.0)\end{array}$ & 0.5 \\
\hline $\begin{array}{c}\text { Major } \\
\text { Diagnostic } \\
\text { Category } \\
\text { (MDC) }\end{array}$ & $\begin{array}{c}\text { Circulatory system } \\
\text { Musculoskeletal system \& } \\
\text { connective tissue } \\
\text { Nervous system } \\
\text { Hepatobiliary/pancreas } \\
\text { Respiratory system } \\
\text { Infectious \& parasitic } \\
\text { Kidney \& urinary tract } \\
\text { Endocrine/nutrition/metabolic } \\
\text { Ear, nose, mouth, and throat } \\
\text { Female reproductive system } \\
\text { Skin, subcutaneous tissue, } \\
\text { breast } \\
\text { Other (MDCs with } \leq 2 \% \\
\text { occurrence) }\end{array}$ & $\begin{array}{c}29,904(25.4) \\
12,521(10.6) \\
8767(7.4) \\
7368(6.3) \\
7094(6.0) \\
5762(4.9) \\
5474(4.6) \\
4207(3.6) \\
2859(2.4) \\
2809(2.4) \\
2459(2.1)\end{array}$ & $\begin{array}{l}1329(8.4) \\
1223(7.7) \\
1190(7.5) \\
1327(8.4) \\
723(4.6) \\
513(3.2) \\
319(2.0) \\
259(1.6) \\
236(1.5)\end{array}$ & $\begin{array}{c}25,974(25.4) \\
11,230(11.0) \\
7438(7.3) \\
6145(6.0) \\
5904(5.8) \\
4435(4.3) \\
4751(4.7) \\
3694(3.6) \\
2540(2.5) \\
2550(2.5) \\
2223(2.2) \\
25,182(24.7)\end{array}$ & $<0.001$ \\
\hline $\begin{array}{c}\text { Overall length } \\
\text { of stay at } \\
\text { hospital }\end{array}$ & $\begin{array}{c}\leq 5 \text { days } \\
5-12 \text { days } \\
12-42 \text { days } \\
>42 \text { days }\end{array}$ & $\begin{array}{c}52,087(44.2) \\
35,210(29.9) \\
26,753(22.7) \\
3834(3.3)\end{array}$ & $\begin{array}{c}5410(34.2) \\
4876(30.8) \\
4482(28.3) \\
1050(6.6)\end{array}$ & $\begin{array}{c}46,677(45.7) \\
30,334(29.7) \\
22,271(21.8) \\
2784(2.7)\end{array}$ & $<0.001$ \\
\hline $\begin{array}{l}\text { Length of stay } \\
\text { by hospital unit }\end{array}$ & $\begin{array}{c}\leq 24 \mathrm{~h} \\
1-3 \text { days } \\
3-7 \text { days } \\
>7 \text { days }\end{array}$ & $\begin{array}{c}52,932(44.9) \\
35,748(30.3) \\
20,916(17.7) \\
8288(7.0)\end{array}$ & $\begin{array}{c}6699(42.4) \\
4865(30.8) \\
2833(17.9) \\
1421(9.0)\end{array}$ & $\begin{array}{c}46,233(45.3) \\
30,883(30.3) \\
18,083(17.7) \\
6867(6.7)\end{array}$ & $<0.001$ \\
\hline $\begin{array}{l}\text { Length of stay } \\
\text { in the ICU }\end{array}$ & $\begin{array}{c}\leq 24 \mathrm{~h} \\
1-3 \text { days } \\
3-7 \text { days } \\
>7 \text { days }\end{array}$ & $\begin{array}{c}2805(28.8) \\
4048(41.6) \\
1928(19.8) \\
947(9.7)\end{array}$ & $\begin{array}{c}198(27.1) \\
322(44.1) \\
134(18.4) \\
76(10.4)\end{array}$ & $\begin{array}{c}2607(29.0) \\
3726(41.4) \\
1794(19.9) \\
871(9.7)\end{array}$ & 0.36 \\
\hline
\end{tabular}

* Some patients appeared in both training and test sets because the data were split on bed movements, not patients. 
Performance of Machine Learning Models at $6 \mathrm{~h}$ Prior to Escalation

The model results are shown in Table 2. At $6 \mathrm{~h}$ prior to escalation, classical MEWS using a cutoff score of 2 had sensitivity of $64.5 \%$, specificity of $66.6 \%$ and AUC of 0.67 (Table 2). MEWS++ (the RF model) had the best performance of all ML models and performed significantly better than classical MEWS, with sensitivity of $81.6 \%$, specificity of $75.5 \%$, and AUC of 0.85 (Table 2 and Figure 2). PPV also improved, with an AUPRC of 0.39 (Table 2 and Figure 2). Interestingly, while the difference between the ROC curves of linear SVM and the RF model was not significant $(p=0.16)$, superior performance of RF was seen by comparing the computed AUCPR and visual inspection of the precision recall (PR) curves (Figure 2). The AUCPR for the RF model was 36.2\% vs. $28.7 \%$ for linear SVM, with no overlap of the $95 \%$ confidence intervals (Table 2 ).

Table 2. Model performance metrics.

\begin{tabular}{|c|c|c|c|c|c|c|c|c|}
\hline Model & $\begin{array}{l}\text { Sensitivity, \% } \\
(95 \% \text { CI })\end{array}$ & $\begin{array}{c}\text { Specificity, \% } \\
(95 \% \text { CI) }\end{array}$ & $\begin{array}{c}\text { Accuracy, \% } \\
(95 \% \text { CI) }\end{array}$ & $\begin{array}{l}\text { PPV, \% } \\
(95 \% \text { CI) }\end{array}$ & F1 Score & $\begin{array}{c}\text { ROC } \\
(95 \% \text { CI })\end{array}$ & $\begin{array}{l}\text { AUC PR } \\
(95 \% \text { CI })\end{array}$ & $p$-Value * \\
\hline $\begin{array}{l}\text { Random Forest } \\
(\mathrm{MEWS}++)\end{array}$ & $\begin{array}{c}78.9 \\
(77.6-80.1)\end{array}$ & $\begin{array}{c}79.1 \\
(78.9-79.3)\end{array}$ & $\begin{array}{c}79.1 \\
(78.9-79.3)\end{array}$ & $\begin{array}{c}11.5 \\
(11.1-11.9)\end{array}$ & 0.2 & $\begin{array}{c}87.9 \\
(87.4-88.4)\end{array}$ & $\begin{array}{c}36.2 \\
(34.7-37.7)\end{array}$ & $<0.0001$ \\
\hline Linear SVM & $\begin{array}{c}79.0 \\
(77.6-80.3)\end{array}$ & $\begin{array}{c}77.9 \\
(77.6-78.1)\end{array}$ & $\begin{array}{c}77.9 \\
(77.7-78.2)\end{array}$ & $\begin{array}{c}11.0 \\
(10.6-11.4)\end{array}$ & 0.19 & $\begin{array}{c}87.3 \\
(86.8-87.9)\end{array}$ & $\begin{array}{c}28.7 \\
(27.2-30.2)\end{array}$ & $<0.00010 .16^{* *}$ \\
\hline LR & $\begin{array}{c}61.4 \\
(59.8-63.0)\end{array}$ & $\begin{array}{c}78.5 \\
(78.3-78.8)\end{array}$ & $\begin{array}{c}77.9 \\
(77.7-78.2)\end{array}$ & $\begin{array}{c}9.0 \\
(8.6-9.4)\end{array}$ & 0.16 & $\begin{array}{c}79.1 \\
(78.4-79.8)\end{array}$ & $\begin{array}{c}17.2 \\
(16.0-18.5)\end{array}$ & $<0.0001$ \\
\hline MEWS Score & $\begin{array}{c}64.2 \\
(62.7-65.7)\end{array}$ & $\begin{array}{c}66.2 \\
(66.0-66.5)\end{array}$ & $\begin{array}{c}66.2 \\
(65.9-66.4)\end{array}$ & $\begin{array}{c}6.1 \\
(5.9-6.4)\end{array}$ & 0.11 & $\begin{array}{c}66.7 \\
(65.9-67.6)\end{array}$ & $\begin{array}{c}7.0 \\
(6.2-7.8)\end{array}$ & \\
\hline
\end{tabular}

${ }^{*} p$-value for difference between AUC ROC for respective ML model and MEWS Score. ${ }^{* *} p$-value $=0.16$ for Random Forest vs. Linear SVM. AUCPR-Area Under Precision Recall Curve, LR-Linear Regression, SVM-Support Vector Machine, ROC-Receiver Operating Characteristic.

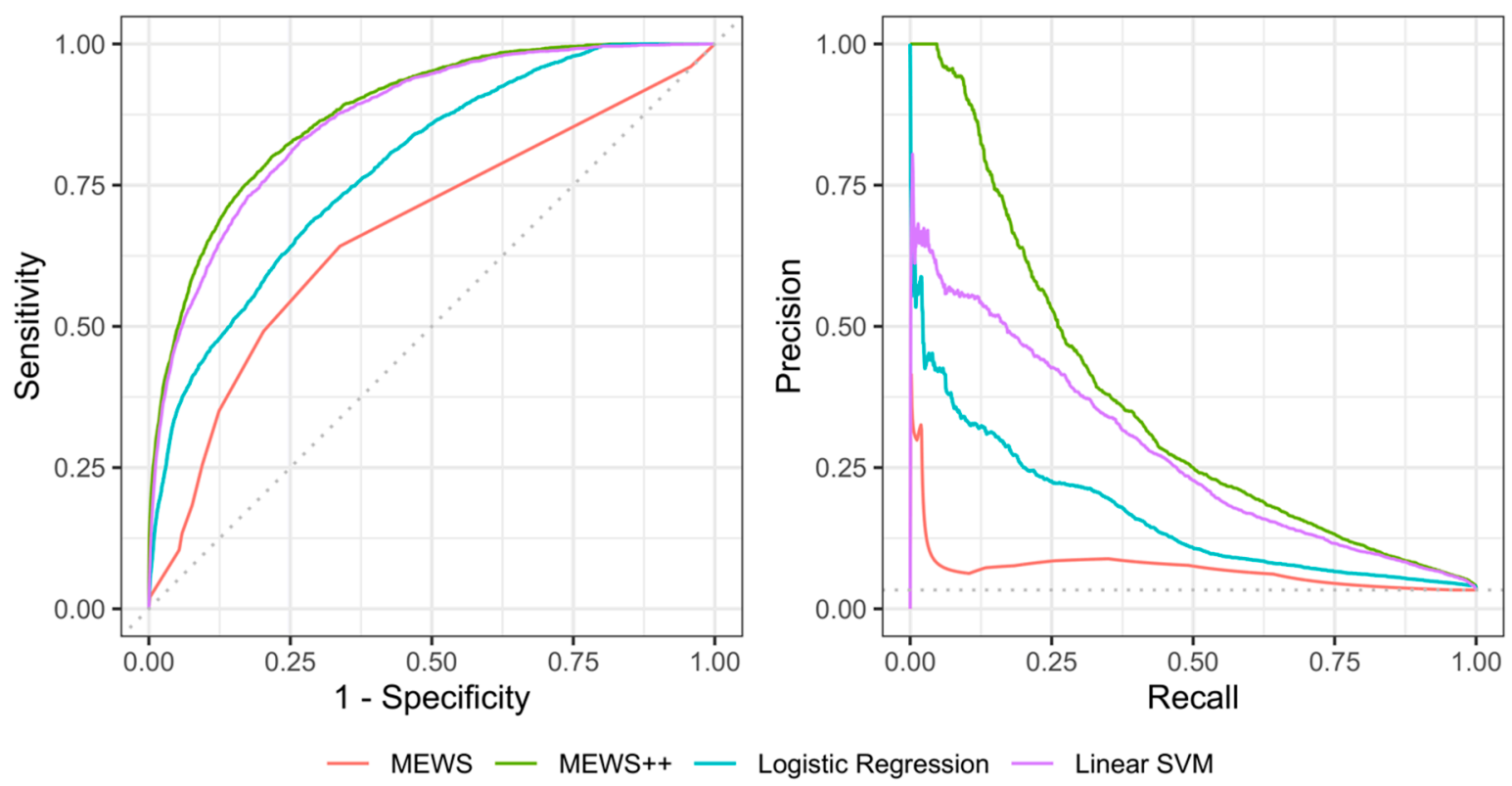

Figure 2. ROC and AUC PR Curves. Receiver Operating Characteristic (ROC) curves (left panel) and Precision-Recall curves (right panel) for the four models evaluated. MEWS++ (RF) performs better than other algorithms. LR-Logistic Regression, SVM = Support Vector Machine.

The 24-h performance of MEWS++ vs. classical MEWS is shown in Figure 3. As can been seen, the RF model is stable over time, whereas the sensitivity of classical MEWS declined as the prediction time prior to escalation increased. 

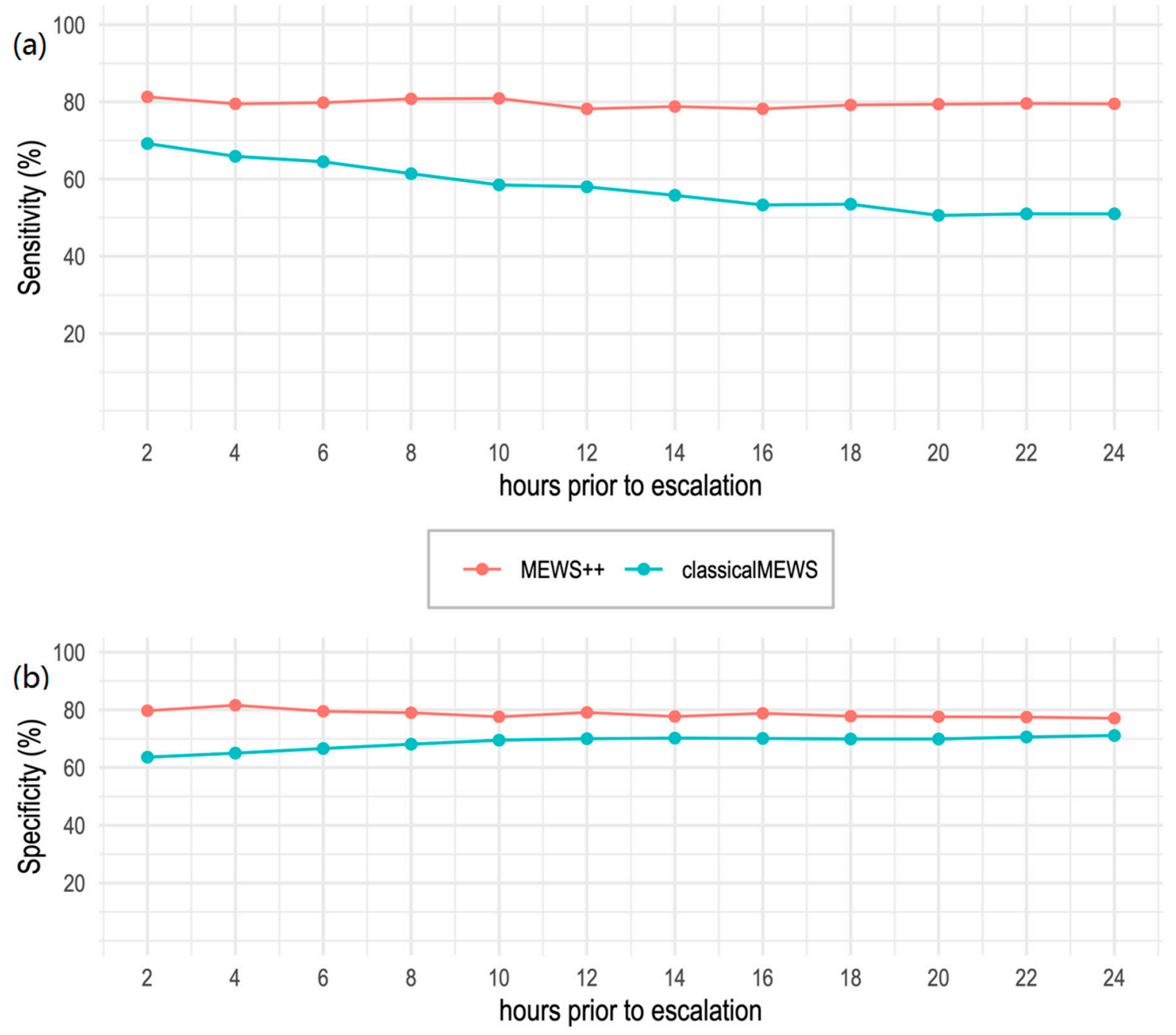

Figure 3. Comparison of 24-h performance of RF Model (MEWS++) vs. classical MEWS. Predictions were generated every $2 \mathrm{~h}$ for $24 \mathrm{~h}$ prior to escalation. A threshold of 2 was used for MEWS, and 0.5 (the default) for the RF model. (a) Sensitivity of MEWS begins to degrade after $4 \mathrm{~h}$ whereas sensitivity of MEWS++ remains stable. (b) Specificity of MEWS++ is consistently higher than MEWS.

Since different MDCs showed different rates of unexpected escalation, a subgroup analysis was performed for the RF model for different MDCs. The five MDCs in which MEWS++ showed the best performance were Respiratory, Infectious, Digestive, Hepatobiliary/Pancreas, and Cardiovascular Diseases (Supplementary Figure S3).

\section{Discussion}

In this retrospective, single center study, we developed a ML model of patient deterioration (MEWS++) that significantly outperformed classical MEWS in predicting, $6 \mathrm{~h}$ in advance, clinical deterioration requiring transfer to a higher acuity unit, or death. The features utilized in the final model were variables that are readily available in all patient care areas, consisting mostly of demographics, vitals, lab results and physical exam findings.

MEWS++ improves on classical MEWS in several ways. The original MEWS algorithm was developed to identify patients at imminent risk of clinical deterioration, using a limited set of features [6]. We used a greatly expanded feature set, continuous value assessment that makes no a-priori assumptions about what constitutes a "normal" range, and time series forecasting where not only "point in time" measurements, but also prior data and trends, are incorporated into every 
prediction. The RF model demonstrated stable performance across a wide prediction window from $2 \mathrm{~h}$ to up to $24 \mathrm{~h}$ prior to the escalation of care. The chosen 6-h window gives enough time for intervention without straining clinical credibility. Similar to the "golden hour principle" that has been applied to a number of clinical conditions, including acute coronary syndrome, stroke and severe sepsis, a 6-h window could enable timely evidence-based interventions that improve outcomes for these patients [28-30].

One challenge with using ML models for clinical prediction is model interpretability [31,32]. It is desirable to present clinicians with a list of most important features. Such a list may build trust in the algorithm as well as provide clinical context for potential action. Ranking features by their Gini coefficient is one approach that can be applied to RF models [27,33]. The top features for MEWS++ are shown in Supplementary Figure S4. When applied to an individual patient, the list of features will vary. For example, the top 10 most important features for patient A may be pulse, BUN, age, systolic BP, bilirubin, diastolic BP, respiration rate, sodium, and lactate. The prediction for Patient B might have a very similar set of most important features, but instead of lactate have the INR. Thus, the features give individualized insight into why a prediction score might be high for a given patient.

Our approach shares similarity with the index developed by Rothman et al [8]. We used similar input data - nursing flowsheets, labs, vitals, and so forth—and similar sampling methodology. Where our approach differs was in the clinical endpoint used for model development and validation (escalation of care/death on the floor vs. 1-year mortality), and the algorithm employed (RF vs. LR). Although the Rothman index showed good performance for an LR model, we found that in our case the RF model performed better than LR. This may have been because of the large numbers of features [34].

While ML approaches based on regression and SVM have been evaluated to detect patient deterioration using only vital signs or in combination with laboratory test results, we found that an RF model performed better than SVM for our use case, with a much higher AUCPR $(36.2 \% \mathrm{vs}$. $28.7 \%$ ) [35-38]. This finding could be explained by the ability of RF to provide a non-parametric, hard to over-train model which is relatively robust to outliers and noise [39]. Therefore, we felt that the RF-based model was most suitable for this study.

\section{Limitations}

There are several limitations to our approach. This was a single center, retrospective study. Our results may not be replicable at other institutions with different patient populations. The phenotyping algorithm used to identify unexpected escalations is based on purely administrative data with no clinical context. The algorithm cannot distinguish between an appropriate escalation to an ICU versus an unplanned emergent transfer-an example of the Frame Problem [40]. The assumption that bed movements are independent events and that prior bed movements do not influence future bed movements may be incorrect. Thus, our phenotype is not a perfect gold standard. This bias in the phenotyping is carried forward into the ML classifier, although the model does attenuate the bias. Also, we did not calibrate the prediction threshold [41,42]. Finally, another limitation of using ML methods is that scores become hard to interpret, as opposed to linear models where determining the contributors to a positive result are easily conceptualized [31]. Nonetheless, ML models may augment human intuition by finding hidden patterns in large datasets.

\section{Conclusions}

Using ML and a large database we have developed a predictive model called MEWS++ that has significantly better performance than the classic MEWS. MEWS++ can warn of patient deterioration $6 \mathrm{~h}$ prior to the event and thus help clinicians make timely interventions. Future models could be improved by incorporating additional data, such as more laboratory results, fluid intake and output, medication data, and free text from provider notes. Looking ahead, the success of using an ML model such as the one developed here as a clinical tool is contingent on its proper integration into healthcare 
system workflows. This work will require multidisciplinary collaboration between data scientists, clinicians, and hospital administrators, in order to fully realize the goal of improved clinical care.

Supplementary Materials: The following are available online at http://www.mdpi.com/2077-0383/9/2/343/s1, Figure S1: Sensitivity and Specificity of Classical MEWS at Different Thresholds; Figure S2: Rate of Escalation by MDC; Figure S3: Sub-group analysis of MEWS++ Performance by Major Diagnostic Category (MDC); Figure S4: Top Features contributing to model, ranked by Gini coefficient; Table S1: Phenotyping Rules; Table S2: Model Parameters; Table S3: Initial List of Features (Variables); Table S4: Final List of 36 Features (Variables) Chosen by Recursive Feature Elimination; Table S5: Results of 10-fold under-sampling of training data on final RF model performance.

Author Contributions: Conceptualization, A.K., P.T., R.M.F., D.L.R. and M.A.L.; Methodology, A.K., P.T., R.M.F. and M.A.L.; Software, M.S.T., A.K. and P.T.; Validation, M.S.T., R.M.F. and M.A.L.; Formal Analysis, A.K., H.N.J. and M.M.; Investigation, Not-applicable; Resources, D.L.R., J.T.D., R.K.-S. and M.M.; Data Curation, A.K., P.T., R.M.F. and M.A.L.; Writing-Original Draft Preparation, A.K. and M.A.L.; Writing-Review \& Editing, D.L.R., R.M.F., R.R.G., E.K., R.K.-S. and M.M.; Visualization, A.K. and H.N.J.; Supervision, J.T.D., M.M. and M.A.L.; Project Administration, R.M.F. and M.M.; Funding Acquisition, Not Applicable. All authors have read and agree to the published version of the manuscript.

Funding: Arash Kia and Prem Timsina were supported by grant UL1TR001433 from the National Center for Advancing Translational Sciences, National Institutes of Health. The remaining authors were supported by departmental funds.

Acknowledgments: This work was supported in part through the computational resources and staff expertise provided by Scientific Computing at the Icahn School of Medicine at Mount Sinai.

Conflicts of Interest: The authors declare no conflicts of interest.

\section{References}

1. Alam, N.; Hobbelink, E.L.; van Tienhoven, A.J.; van de Ven, P.M.; Jansma, E.P.; Nanayakkara, P.W. The impact of the use of the Early Warning Score (EWS) on patient outcomes: A systematic review. Resuscitation 2014, 85, 587-594. [CrossRef]

2. Brown, H.; Terrence, J.; Vasquez, P.; Bates, D.W.; Zimlichman, E. Continuous monitoring in an inpatient medical-surgical unit: A controlled clinical trial. Am. J. Med. 2014, 127, 226-232. [CrossRef]

3. Buist, M.; Bernard, S.; Nguyen, T.V.; Moore, G.; Anderson, J. Association between clinically abnormal observations and subsequent in-hospital mortality: A prospective study. Resuscitation 2004, 62, $137-141$. [CrossRef]

4. Hillman, K.M.; Bristow, P.J.; Chey, T.; Daffurn, K.; Jacques, T.; Norman, S.L.; Bishop, G.F.; Simmons, G. Antecedents to hospital deaths. Intern. Med. J. 2001, 31, 343-348. [CrossRef]

5. McNeill, G.; Bryden, D. Do either early warning systems or emergency response teams improve hospital patient survival? A systematic review. Resuscitation 2013, 84, 1652-1667. [CrossRef]

6. Subbe, C.P.; Kruger, M.; Rutherford, P.; Gemmel, L. Validation of a modified Early Warning Score in medical admissions. QJM 2001, 94, 521-526. [CrossRef] [PubMed]

7. Tarassenko, L.; Hann, A.; Young, D. Integrated monitoring and analysis for early warning of patient deterioration. Br. J. Anaesth. 2006, 97, 64-68. [CrossRef] [PubMed]

8. Rothman, M.J.; Rothman, S.I.; Beals, J. Development and validation of a continuous measure of patient condition using the Electronic Medical Record. J. Biomed. Inform. 2013, 46, 837-848. [CrossRef] [PubMed]

9. Bliss, J.P.; Gilson, R.D.; Deaton, J.E. Human probability matching behaviour in response to alarms of varying reliability. Ergonomics 1995, 38, 2300-2312. [CrossRef] [PubMed]

10. Cvach, M. Monitor alarm fatigue: An integrative review. Biomed. Instrum. Technol. 2012, 46, $268-277$. [CrossRef]

11. Fleischman, W.; Ciliberto, B.; Rozanski, N.; Parwani, V.; Bernstein, S.L. Emergency department monitor alarms rarely change clinical management: An observational study. Am. J. Emerg. Med. 2019, 158370. [CrossRef] [PubMed]

12. Bonafide, C.P.; Lin, R.; Zander, M.; Graham, C.S.; Paine, C.W.; Rock, W.; Rich, A.; Roberts, K.E.; Fortino, M.; Nadkarni, V.M.; et al. Association between exposure to nonactionable physiologic monitor alarms and response time in a children's hospital. J. Hosp. Med. 2015, 10, 345-351. [CrossRef] [PubMed] 
13. Bedoya, A.D.; Clement, M.E.; Phelan, M.; Steorts, R.C.; O'brien, C.; Goldstein, B.A. Minimal Impact of Implemented Early Warning Score and Best Practice Alert for Patient Deterioration. Crit. Care Med. 2019, 47, 49-55. [CrossRef] [PubMed]

14. Hu, S.B.; Wong, D.J.L.; Correa, A.; Li, N.; Deng, J.C. Prediction of Clinical Deterioration in Hospitalized Adult Patients with Hematologic Malignancies Using a Neural Network Model. PLoS ONE 2016, 11, e0161401. [CrossRef]

15. Rubin, J.; Potes, C.; Xu-Wilson, M.; Dong, J.; Rahman, A.; Nguyen, H.; Moromisato, D. An ensemble boosting model for predicting transfer to the pediatric intensive care unit. Int. J. Med. Inform. 2018, 112, 15-20. [CrossRef]

16. Wikipedia contributors: Major Diagnostic Category. Wikipedia, the Free Encyclopedia. 2017. Available online: https://en.wikipedia.org/w/index.php?title=Major_Diagnostic_Category\&oldid=793887852 (accessed on 22 March 2019).

17. Mathukia, C.; Fan, W.; Vadyak, K.; Biege, C.; Krishnamurthy, M. Modified Early Warning System improves patient safety and clinical outcomes in an academic community hospital. J. Community Hosp. Intern. Med. Perspect. 2015, 5. [CrossRef]

18. Ludikhuize, J.; de Jonge, E.; Goossens, A. Measuring adherence among nurses one year after training in applying the Modified Early Warning Score and Situation-Background-Assessment-Recommendation instruments. Resuscitation 2011, 82, 1428-1433. [CrossRef]

19. Yu, S.; Ma, Y.; Gronsbell, J.; Cai, T.; Ananthakrishnan, A.N.; Gainer, V.S.; Churchill, S.E.; Szolovits, P.; Murphy, S.N.; Kohane, I.S.; et al. Enabling phenotypic big data with PheNorm. J. Am. Med. Inform. Assoc. 2018, 25, 54-60. [CrossRef]

20. Hastie, T.; Tibsharani, R.; Friedman, J. The Elements of Statistical Learning: Data Mining, Inference, and Prediction, 2nd ed.; Springer Science+Business Media: New York, NY, USA, 2009; Available online: http: //www.springerlink.com/index/D7X7KX6772HQ2135.pdf (accessed on 15 May 2018).

21. Hsieh, C.-J.; Chang, K.-W.; Lin, C.-J.; Keerthi, S.S.; Sundararajan, S. A Dual Coordinate Descent Method for Large-Scale Linear SVM. In Proceedings of the 25th International Conference on Machine Learning-ICML '08, July 2008; pp. 408-415. Available online: http://portal.acm.org/citation.cfm?doid=1390156.1390208 (accessed on 15 May 2018).

22. Fan, R.-E.; Chang, K.-W.; Hsieh, C.-J.; Wang, X.R.; Lin, C.J. LIBLINEAR: A Library for Large Linear Classification. J. Mach. Learn. Res. 2008, 9, 1871-1874.

23. Maniruzzaman, M.; Rahman, M.J.; Al-MehediHasan, M.; Suri, H.S.; Abedin, M.M.; El-Baz, A.; Suri, J.S. Accurate Diabetes Risk Stratification Using Machine Learning: Role of Missing Value and Outliers. J. Med. Syst. 2018, 42, 92. [CrossRef]

24. Spark 2.4.0 ScalaDoc-MinMaxScaler. Available online: http://spark.apache.org/docs/latest/api/scala/index. html\#org.apache.spark.ml.feature.MinMaxScaler (accessed on 28 November 2018).

25. Krawczyk, B. Learning from imbalanced data: Open challenges and future directions. Prog. Artif. Intell. 2016, 5, 221-232. [CrossRef]

26. The Apache Software Foundation: MLlib: Main Guide-Spark 2.3.0 Documentation. spark.apache.org 2018. Available online: https://spark.apache.org/docs/latest/ml-guide.html (accessed on 22 March 2019).

27. Yin, Z.; Zhang, J. Operator functional state classification using least-square support vector machine based recursive feature elimination technique. Comput. Methods Programs Biomed. 2014, 113, 101-115. [CrossRef] [PubMed]

28. Wheeler, D.S. Is the "golden age" of the "golden hour" in sepsis over? Crit. Care 2015, 19, 10-12. [CrossRef] [PubMed]

29. Ebinger, M.; Kunz, A.; Wendt, M.; Rozanski, M.; Winter, B.; Waldschmidt, C.; Weber, J.; Villringer, K.; Fiebach, J.B.; Audebert, H.J. Effects of golden hour thrombolysis: A Prehospital Acute Neurological Treatment and Optimization of Medical Care in Stroke (PHANTOM-S) substudy. JAMA Neurol. 2015, 72, 25-30. [CrossRef]

30. Clark, D.E. RA Cowley, the "Golden Hour", the "Momentary Pause", and the "Third Space". Am. Surg. 2007, 83, 1401-1406.

31. Beam, A.L.; Kohane, I.S. Big data and machine learning in health care. J. Am. Med. Assoc. 2018, 319, 1317-1318. [CrossRef] 
32. Elshawi, R.; Al-Mallah, M.H.; Sakr, S. On the interpretability of machine learning-based model for predicting hypertension. BMC Med. Inform. Decis. Mak. 2019, 19, 146. [CrossRef]

33. Palczewska, A.; Palczewski, J.; Robinson, R.M.; Neagu, D. Interpreting random forest models using a feature contribution method. In Proceedings of the IEEE Conference on Information Reuse and Integration, San Francisco, CA, USA, 14-16 August 2013; pp. 112-119. Available online: https://ieeexplore.ieee.org/ document/6642461 (accessed on 15 May 2018).

34. Couronné, R.; Probst, P.; Boulesteix, A.L. Random forest versus logistic regression: A large-scale benchmark experiment. BMC Bioinform. 2018, 19, 270. [CrossRef]

35. Clifton, L.; Clifton, D.A.; Watkinson, P.J.; Tarassenko, L. Identification of patient deterioration in vital-sign data using one-class support vector machines. In Proceedings of the 2011 Federated Conference on Computer Science and Information Systems, Szczecin, Poland, 18-21 September 2011; pp. 125-131.

36. Tarassenko, L.; Clifton, D.A.; Pinsky, M.R.; Hravnak, M.T.; Woods, J.R.; Watkinson, P.J. Centile-based early warning scores derived from statistical distributions of vital signs. Resuscitation 2011, 82, 1013-1018. [CrossRef]

37. Bai, Y.; Do, D.H.; Harris, P.R.E.; Schindler, D.; Boyle, N.G.; Drew, B.J.; Hu, X. Integrating monitor alarms with laboratory test results to enhance patient deterioration prediction. J. Biomed. Inform. 2015, 53, 81-92. [CrossRef]

38. Escobar, G.J.; Laguardia, J.C.; Turk, B.J.; Ragins, A.; Kipnis, P.; Draper, D. Early detection of impending physiologic deterioration among patients who are not in intensive care: Development of predictive models using data from an automated electronic medical record. J. Hosp. Med. 2012, 7, 388-395. [CrossRef] [PubMed]

39. Touw, W.G.; Bayjanov, J.R.; Overmars, L.; Backus, L.; Boekhorst, J.; Wels, M.; van Hijum, S.A. Data mining in the Life Sciences with Random Forest: A walk in the park or lost in the jungle? Brief. Bioinform. 2013, 14, 315-326. [CrossRef] [PubMed]

40. Yu, K.H.; Kohane, I.S. Framing the challenges of artificial intelligence in medicine. BMJ Qual. Saf. 2019, 28, 238-241. [CrossRef] [PubMed]

41. Baker, T.; Gerdin, M. The clinical usefulness of prognostic prediction models in critical illness. Eur. J. Intern. Med. 2017, 45, 37-40. [CrossRef]

42. Steyerberg, E.W.; Vickers, A.J.; Cook, N.R.; Gerds, T.; Gonen, M.; Obuchowski, N.; Kattan, M.W. Assessing the Performance of Prediction Models. Epidemiology (Cambridge Massachusetts) 2010, 21, 128-138. [CrossRef] 